\title{
Software
}

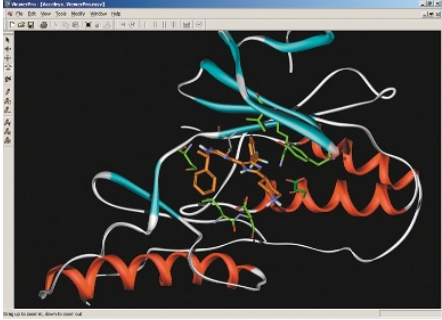

\section{Integrated applications}

The Discovery Studio software platform from Accelrys is designed to help reduce the time and cost involved in the discovery and development of novel drugs and therapies. Its Windows-based bioinformatics and chemistry applications include Discovery Studio Gene for viewing, editing, and analyzing DNA and protein sequences; Discovery Studio ViewerPro, a molecular modeling and visualization tool; and Discovery Studio MedChem Explorer, for pharmacophore modeling and design. These applications can be integrated through Discovery Studio Project Knowledge Manager, an Oracle-based groupware infrastructure that captures and stores the information and knowledge generated and facilitates effective reuse across research teams. http://www.accelrys.com

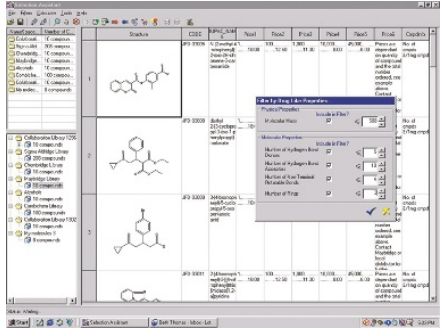

\section{Compound selection}

IDBS offers Selection Assistant, an application for facilitating the browsing, filtering, editing, and registration of chemical information sourced from structure data (SD) files, the industry standard electronic format for communicating chemical compound data. Users can view and edit structures and their properties, including associated data such as price, availability, and purity of a compound. The application supports threedimensional visualization of structures through links to ChemDraw and ISISDraw. Selection Assistant can be deployed on a stand-alone basis, or in conjunction with IDBS's StructureBase enterprise application for chemical registration and searching. http://www.id-bs.com

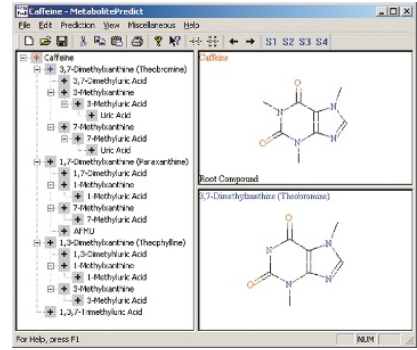

\section{Data for drugs}

Bruker Daltonics' MetaboliteTools includes several modules for the automated prediction, detection, and identification of drug metabolites based on the chemical structure of the parent drug. It can be used for detailed compound characterization and structure elucidation, as well as high-throughput automated metabolite detection. MetaboliteTools can be used interactively with the acquisition software of Bruker Daltonics' Esquire ion trap systems for intelligent data acquisition feedback in directed LC-MS-MS experiments. http://www.bdal.de/metabolite.html

\section{Purification made simple}

The Purify Easy Access software from Agilent Technologies helps chemists analyze and purify large numbers of organically synthesized drug candidates by simplifying access to the company's LC-MS systems. Users can simply walk up with their samples, input basic sample information, choose an appropriate purification scheme, load their samples, and then return to their labs. Email notification tells them when purification of their samples has been completed. Complete system and sample information is always available including current status, estimated time to complete all the studies in the queue, estimated time to complete the current study, and color-coded status of individual samples and fractions in a well plate.

http://www.agilent.com

\section{Sequence assembly}

Paracel's GenomeAssembler is a complete software package for all types of sequence assembly, including whole-genome shotgun projects. It performs all of the steps required for sequence reconstruction including base calling, contaminant screening, constraints generation, and assembly. When starting with raw trace files and base calling is needed, the software uses Paracel's proprietary TraceTuner technology to call the bases. GenomeAssembler controls the different

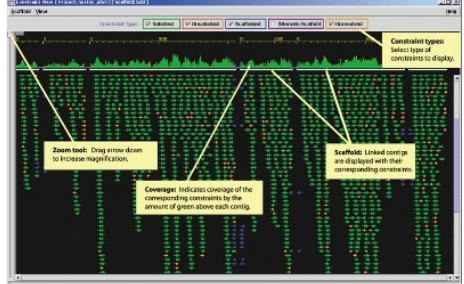

steps in the pipeline via a simple graphical user interface or command line. GenomeAssembler also can assist during the finishing phases of an assembly project, offering viewing and editing capabilities for contigs, scaffolds, and corresponding constraints. http://www.paracel.com

\section{SiRNA design}

DNAengine's OligoEngine software helps researchers determine the optimum sequence and structure of small interfering RNA (siRNA) duplexes for RNA interference applications. Researchers can log on to the OligoEngine website, use the software to choose the most effective siRNA duplex design for their work, and then order those sequences directly from OligoEngine. The process is seamlessly integrated for quick and easy purchasing, and every siRNA molecule is backed by OligoEngine's quality guarantee. http://www.oligoengine.com

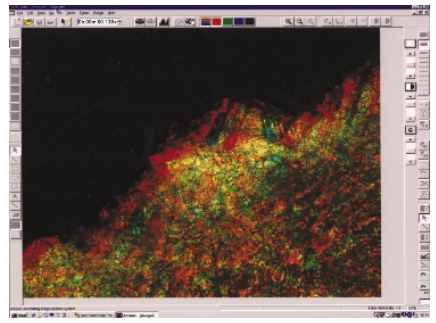

\section{Image capture system}

AcQuis Version 2.0 is Syncroscopy's updated image documentation and archiving system, designed to make capturing images of samples stained with colored or fluorescent dyes both quicker and more accurate. It can be used with an RGB camera to display and acquire a separate red, blue, and green image in sequence using the new AcQuis Multiband technique, which allows users to set the exposure and other conditions for each charge-coupled device channel and produce a perfectly balanced image from their microscopes in the shortest time possible - an advantage when using fluorescent dyes that fade rapidly.

http://www.syncroscopy.com 